\title{
Victimización en la escuela, ocio digital e irritabilidad: análisis mediante ecuaciones estructurales
}

\author{
Victimization in school, digital leisure and irritability: analysis using structural equations
}

\author{
Chacón-Cuberos, Ramón ${ }^{(1)}$; Castro-Sánchez, Manuel ${ }^{(2)}$; González-Campos, Gloria ${ }^{(3)}$ \& \\ Zurita-Ortega, Félix ${ }^{(4)}$
}

(1) Universidad de Huelva (2) Universidad de Almería (3) Universidad de Sevilla (4) Universidad de Granada

\begin{abstract}
Victimization in school represents a worrying phenomenon given the negative consequences associated with it, such as states of depression or anxiety which are related to digital leisure habits. This descriptive and cross-sectional study, which was conducted on a sample of 1038 students of third cycle of Primary Education $(\mathrm{M}=11,33$; $\mathrm{SD}=1,27)$, aims to define and contrast an explanatory model about the use of television and video games based on victimization. The instruments employed were the School Victimization Scale (Mynard \& Joseph, 2000), the Questionnaire of Experiences related to Video games (Chamarro et al., 2014) and an ad hoc questionnaire for the registration of variables related to digital leisure. The structural model was correctly developed and with good reliability. The results showed positive relationships between the three types of victimization, physical victimization and use of video games and television, and verbal victimization and use of television. Furthermore, digital leisure habits were positively associated with levels of irritability, which shows how bullying can act as a risk factor in the problematic use of video games and television. Victimization may constitute a risk factor in the pathological consumption of screen leisure and irritability states.
\end{abstract}

Reception Date 2018 June 12

Approval Date

2018 June 28

Publication Date:

2018 June 28

Fecha de recepción

2018 Junio 12

Fecha de aprobación 2018 Junio 28

Fecha de publicación 2018 Junio 28 videojuegos y televisión, y la victimización verbal con el uso de televisión. Los hábit ocio digital se asociaron positivamente con los niveles de irritabilidad de los escolares, lo que muestra como el acoso escolar podría actuar como factor de riesgo en el ocio digital. Las situaciones de victimización pueden constituir un factor de riesgo en el consumo patológico de ocio de pantalla y estados de irritabilidad.

Palabras clave: escolares; acoso escolar; victimización; televisión; videojuegos.

El término acoso escolar o "bullying" hace referencia a las agresiones de tipo multifacético -físicas, psicológicas o relacionales- que se producen de forma prolongada en contextos escolares entre pares, generando una situación de maltrato e 
indefensión que la víctima no puede abandonar por sus propios medios (Hiduja y Patchin, 2010; López y Sabater, 2014). En base a lo expuesto, un constructo íntimamente relacionado con las situaciones de acoso en la escuela es la victimización. Este término es definido por Turner, Shattuck, Finkelhor y Hamby (2017) como aquella condición mediante la cual un sujeto se considera víctima de algún tipo de situación transgresora -abuso físico, violencia familiar, asilamiento social, etc.- la cual genera vulnerabilidad $\mathrm{y}$ consecuencias traumáticas, especialmente cuando se sufre desde edades tempranas como es el caso de la victimización en edad escolar (Hiduja \& Patchin, 2010; Turner et al., 2017).

Dada la expansión de esta situación en los últimos años (Benbenishty, Avi, Roziner, \& Wrabel, 2016), y las consecuencias negativas que se le asocian -baja autoestima, estrés, depresión, ansiedad, suicidio, etc.- (Boxer, Groves, \& Docherty, 2015; Turner, Exum, Brame, \& Holt, 2013), su estudio, prevención $\mathrm{y}$ tratamiento se ha convertido en un tema de capital importancia a nivel internacional. Ante esta perspectiva, estudios como el de Carrasco y Trianes (2015) o Vilches (2015) destacan el papel de la adaptación social y emocional como medio de prevención, pues podría generar su aparición un cierto nivel de inadaptación fruto de malas prácticas de crianza por parte del sector parental o acciones inclusivas deficientes en entorno escolar.

Son múltiples los factores riesgo que pueden asociarse a situaciones de violencia escolar, como son patrones de comportamiento antisocial, menor capacidad de autorregulación, ausencia empática o fracaso escolar en el caso del agresor (Delisi, Vaughn, Gentile, Anderson, \& Shook, 2013; García, Pérez, \& Nebot, 2010), o bien personalidad introvertida o rasgos físicos, cognitivos o culturales distintos en el caso de la víctima (Mishna, Khoury-Kassabri, Gadalla, \& Daciuk, 2012). Del mismo modo, estudios como el de Ferguson, Olson, Kutner, y Warner (2010) o Vallejos y Capa (2010) prueban la relación entre el consumo de televisión y videojuegos de contenido violento y los niveles de agresividad en sujetos, cristalizando en un nuevo factor de riesgo, dada la gran cantidad de horas que los escolares invierten en ocio digital de pantalla (Boxer et al., 2015; Chacón, Castro, Zurita, Espejo \& Martínez, 2016).

Autores como López y Sabater (2014) señalan que los jóvenes pueden ser muy influenciables en sus patrones de comportamiento, especialmente a través de los medios audiovisuales como exosistema. El motivo es que la televisión les ofrece una fuente de imitación de conducta a través del modelo de imitación social, afectando especialmente a aquellos niños y niñas que no han vivenciado un proceso socializador normalizado (Ferguson et al., 2010; López \& Sabater, 2014; Vilches, 2015). En esta línea, Delisi et al. (2013) recuerdan como diversas teorías sobre el comportamiento violento han comprobado que el aprendizaje y los procesos mentales que implican los contenidos violentos multimedia pueden generar comportamientos agresivos posteriores, ya que producen una desensibilización ante situaciones violentas al ser percibidas como un contenido emocional positivo. De hecho, la exposición continuada se relaciona con reducciones psicológicas y fisiológicas del miedo y ansiedad ante la violencia, produciendo consecuencias cognitivas y afectivas como una disminución de la percepción ante la severidad de las lesiones, la atención a eventos violentos, una perdida en la capacidad empática con las víctimas o el desarrollo de actitudes violentas hacia otros (Boxer et al., 2015; Ditrrick, Beran, Mishna, Hetherington \& Shariff, 2013; Hasan, Bègue, Scharkow \& Bushman, 2013).

Un estudio reciente en este campo es el desarrollado por Watt, Fitzpatrick, Derevensky, y Pagani (2015), quienes analizan las asociaciones entre el uso de televisión en niños y niñas en edad escolar y los niveles de victimización que reportaban, obteniendo un aumento del acoso recibido en 
un $11 \%$ en los escolares que más veían la televisión. En una línea similar, Boxer et al. (2015) estudian el efecto negativo que genera el uso de videojuegos en el rendimiento escolar y las habilidades sociales de niños y adolescentes. Asimismo, Martínez, Betancourt y González (2013) encontraron una relación positiva entre la exposición a videojuegos y la sintomatología depresiva y violencia intrafamiliar en jóvenes adolescentes.

En este sentido, se ha demostrado cómo las víctimas de acoso escolar pueden presentar problemas cognitivos ligados en ocasiones a un uso problemático del ocio digital sedentario lo cual constituye la base de este estudio.

\section{Objetivos}

Este estudio plantea como principales objetivos:

a) analizar y establecer relaciones entre los diferentes tipos de victimización y las variables asociados al uso intensivo de videojuegos y televisión.

b) contrastar un modelo de ecuaciones estructurales que permita explicar el ocio digital sedentario en función de los niveles de victimización y sus dimensiones en una muestra de escolares

c) analizar el efecto de la victimización en el consumo de televisión y videojuegos, así como el efecto de estos dispositivos en los niveles de irritabilidad $\mathrm{y}$ en la sustitución de actividades.

\section{Método}

\section{Diseño}

Se realizó un estudio cuantitativo, no experimental, ex post-facto, de carácter descriptivo, exploratorio y corte transversal. Asimismo, se realiza una única medición en un único grupo.

\section{Participantes}

La muestra estuvo compuesta por 1038 escolares de tercer ciclo de Educación Primaria de 9 centros educativos públicos y concertados de la capital de Granada (España), todos ellos seleccionados mediante muestreo aleatorio por conglomerados considerando la tipología de los centros educativos. La distribución según el sexo de los participantes fue del 49,5\% $(n=514)$ para los varones y del 50,5\% $(n=524)$ para las niñas. La edad estuvo comprendida entre los 10 y 13 años de edad $(\mathrm{M}=11,33 ; \mathrm{DT}=$ 1,27).

Tabla 1 - Características de la muestra

\begin{tabular}{lcc}
\hline & $\mathbf{N}$ & $\mathbf{\%}$ \\
\hline Sexo & & \\
\hline Hombre & 514 & 49,5 \\
Mujer & 524 & 50,5 \\
\hline Tipo de centro & & \\
\hline Público & 578 & 55,7 \\
Privado & 460 & 44,3 \\
\hline
\end{tabular}

\section{Instrumentos}

En la presente investigación se han utilizado tres instrumentos, la Escala de Victimización en la Escuela, el Cuestionario de Experiencias Relacionadas con Videojuegos y un cuestionario construido Ad Hoc para el registro de las variables sociodemográficas, como el sexo, la edad y el tipo de centro en el que realizan sus estudios los participantes del estudio.

Escala de Victimización en la Escuela. Este cuestionario fue desarrollado por Mynard y Joseph (2000) y adaptado al castellano por Cava, Musitu, y Murgui (2007). Se compone de 20 ítems puntuados mediante una escala de tipo Likert ( $1=$ Nunca; 4 = Siempre) la cual muestra tres tipos de victimización; Victimización Física (Ítems 3+4+6+11+16+20), Victimización Verbal (Ítems $3+4+6+11+16+20$ ) y Victimización Relacional (Ítems $2+5+7+8+10+12+14+17+18+19)$. En el estudio original, Mynard y Joseph (2000) obtuvieron una consistencia interna (alpha de Cronbach) de $\alpha=0,77$. En la presente investigación se ha obtenido un coeficiente superior con un $\alpha=0,91$.

Cuestionario de Experiencias Relacionadas con Videojuegos (CERV). La 
escala original fue desarrollada por Chamarro et al. (2014) en jóvenes adolescentes. Este instrumento comprende 17 ítems de tipo negativo que son valorados mediante una escala Likert de cuatro opciones de respuesta donde 1 = Casi Nunca y 4 = Casi siempre. Destacar que este cuestionario permite conocer el uso problemático de videojuegos a través de una categorización en terciles de dicha variable. Asimismo, la consistencia interna de esta escala en el presente trabajo de investigación ha sido de $\alpha=0,89$, superior a la presentada por Chamorro et al. (2014) en el trabajo original $(\alpha=0,87)$

Cuestionario construido Ad Hoc. Se emplea para el registro de variables de tipo sociodemográfico (sexo, edad y centro). Asimismo, se incluyen otras variables relacionadas con el ocio digital; televisión (determina el número de horas diarias que los niños y niñas dedican a ver la televisión); irritabilidad (puntúa mediante una escala de tipo Likert del 1 al 10 el sentimiento de mal humor cuando los escolares no pueden realizar ocio digital); sustitución (indica mediante una escala de tipo Likert de 10 opciones hasta qué punto los niños y niñas dejan de hacer otras actividades para jugar con videojuegos o ver la televisión).

\section{Procedimiento de recogida de datos}

Para la realización de la presente investigación y la recogida de datos se comenzó informando a los directores de los centros educativos que aceptaron participar en este estudio mediante una carta informativa elaborada desde el departamento de Didáctica de la Expresión Musical, Plástica y Corporal de la Universidad de Granada. En este documento se detalló la naturaleza del estudio y se solicitó a los centros educativos su participación. Los centros que aceptaron participar en el trabajo de investigación solicitaron el consentimiento informado de los tutores legales del alumnado para su participación en el estudio.

Posteriormente se aplicaron los instrumentos descritos en el apartado anterior en marzo de 2016. Este proceso se realizó en los centros educativos en horario lectivo, contando con la presencia del tutor de los escolares y la de los investigadores, con el fin de asegurar la correcta cumplimentación de los cuestionarios. Se aseguró al alumnado la confidencialidad de sus datos y que estos serían tratados con fines científicos. Se informó a los centros educativos sobre el posterior informe de los resultados del estudio. Se eliminaron un total de 78 cuestionarios por encontrarse mal cumplimentados.

Este trabajo de investigación fue aprobado por el comité de investigación de la Universidad de Granada y ha cumplido las normas éticas para investigación de la Declaración de Helsinki de 1975.

\section{Análisis de datos}

Los estadísticos básicos se realizaron a través del software IBM SPSS ${ }^{\circledR}$ en su versión 22.0, empleando medias, frecuencias y comparación de medias mediante prueba T. Para analizar las relaciones y efectos entre las variables estudiadas en el modelo estructural diseñado se empleó el programa IBM AMOS ${ }^{\circledR}$ 23.0, fijando el intervalo de confianza en el $95 \%$ con una probabilidad de error del 5\%.

A partir de los objetivos diseñados, se desarrolla el supuesto teórico que sustenta este trabajo a través del siguiente modelo hipotético (Figura 1) con los factores; Factor 1: Victimización Relacional (VR), Factor 2: Victimización Física (VF), Factor 3: Victimización Verbal (VV), Factor 4: Horas diarias de televisión (Televisión), Factor 5: Uso problemático de videojuegos (Videojuegos), Factor 6: Nivel de irritabilidad por no realizar ocio digital (Irritabilidad), Factor 7: Sustitución de otros actividades por ocio digital (Sustitución).

La victimización relacional, victimización física y victimización verbal actúan en el modelo diseñado como variables exógenas, mientras que el uso de televisión, videojuegos, nivel de irritabilidad y sustitución de actividades actúan como variables endógenas (reciben la influencia de otras y necesitan de variables de error de 
predicción). Se emplean flechas bidireccionales (covarianzas) para relacionar las variables exógenas del modelo, mientras que las flechas con una dirección muestran los efectos entre las variables utilizadas (directos e indirectos). Asimismo, se emplea el método de máxima verosimilitud (ML) para la estimación de parámetros, ya que es invariante al tipo de escala.

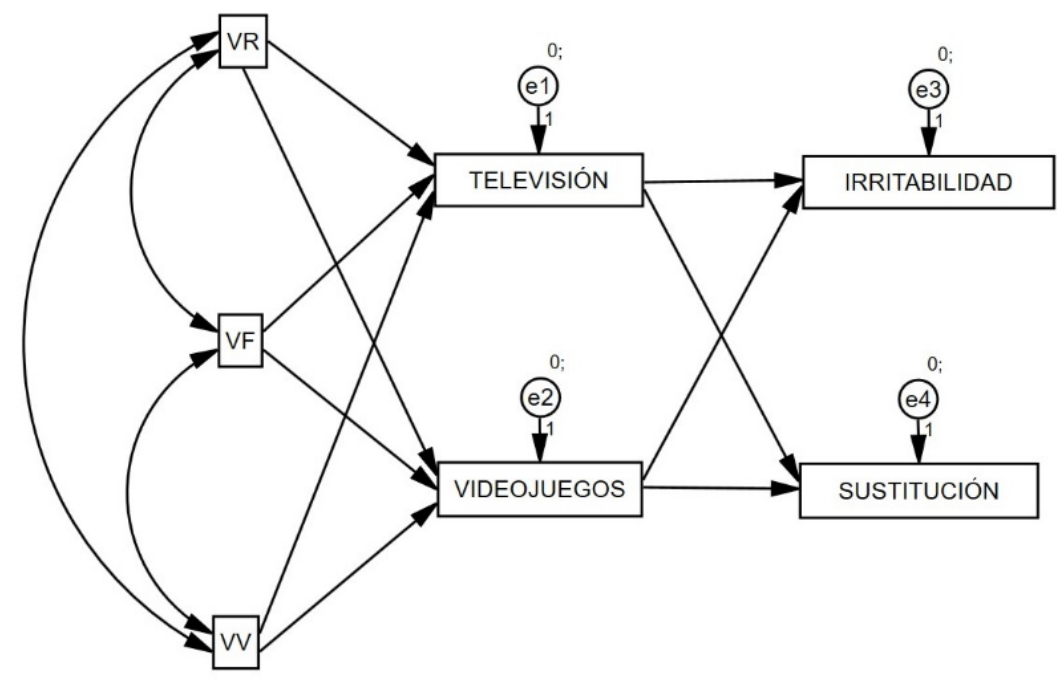

Figura 1. Modelo hipotético de victimización y su relación con el uso de videojuegos y televisión

Se comprobaron los índices de ajuste del modelo estructural con el fin de verificar su compatibilidad y la información empírica obtenida. Se consideraron los criterios de bondad de ajuste de Marsh (2007, p.785) para determinar la fiabilidad de ajuste. De este modo, los valores no significativos para la prueba del Chi-cuadrado se asocian a un buen ajuste. Por otro lado, el índice de ajuste normalizado (NFI) deberá ser superior a 0,90. Asimismo, el valor del índice de ajuste comparativo (CFI) será aceptable si presenta valores superiores a 0,90 y excelente a partir de 0,95. El valor del índice de incremento de ajuste (IFI) será aceptable con coeficientes por encima de 0,90 y excelente para aquellos superiores a 0,95. Finalmente, el valor del error cuadrático medio de aproximación (RMSEA) será excelente si es inferior a 0,05 y aceptable si es inferior a 0,08 .

\section{Resultados}

La Tabla 2 muestra los descriptivos básicos de las variables estudiadas en la muestra según el sexo de la misma. En relación a la victimización relacional, se muestra un valor medio de $1,77 \pm 0,59$ para los varones y de 1,73 $\pm 0,59$ para las féminas sin encontrar diferencias significativas. En el caso de la victimización física si se observan diferencias estadísticamente significativas ( $\mathrm{p}$ $=0,009$ ), mostrando los niños un mayor valor medio que las niñas $(1,68 \pm 0,66$ vs. $1,54 \pm$ $0,55)$. Tampoco se observan diferencias estadísticas para la victimización verbal, con un valor medio de 1,96 $\pm 0,67$ para el sexo masculino y de 1,94 $\pm 0,67$ para el femenino. Por otro lado, se observaron diferencias estadísticamente significativas en el uso de videojuegos $(\mathrm{p}=0,001)$ y televisión $(\mathrm{p}=$ 0,044 ), mostrándose en ambos casos un valor medio superior en los varones (36,11 $\pm 7,31$ vs. $33,82 \pm 6,78$ y $2,94 \pm 1,30$ vs. $2,72 \pm 1,18$, respectivamente). Finalmente, destacar que en ambos sexos obtuvieron el mismo valor en sustitución de actividades $(5,95)$ sin encontrar diferencias estadísticas, mientras que para la irritabilidad $(p=0,001)$ se observan valores más elevados para el sexo masculino $(7,05 \pm$ 2,45 vs. 6,40 vs. 2,20 ). 
Chacón-Cuberos, Ramón; Castro-Sánchez, Manuel; González-Campos, Gloria \& Zurita-Ortega, Félix (2018).

Victimización en la escuela, ocio digital e irritabilidad: análisis mediante ecuaciones estructurales. RELIEVE, 24(1), art. 3. doi: http://doi.org/10.7203/relieve.24.1.12614

Tabla 2 - Descriptivos básicos de la muestra según género

\begin{tabular}{|c|c|c|c|c|c|c|}
\hline & Género & Media & DT & Levene (F) & Levene (Sig.) & Prueba T (Sig.) \\
\hline \multirow{2}{*}{$\begin{array}{l}\text { Victimización } \\
\text { relacional }\end{array}$} & Hombre & 1,77 & 0,59 & \multirow{2}{*}{0,224} & \multirow{2}{*}{0,636} & \multirow{2}{*}{0,489} \\
\hline & Mujer & 1,73 & 0,59 & & & \\
\hline \multirow{2}{*}{$\begin{array}{l}\text { Victimización } \\
\text { física }\end{array}$} & Hombre & 1,68 & 0,66 & \multirow{2}{*}{11,955} & \multirow{2}{*}{0,001} & \multirow{2}{*}{0,009} \\
\hline & Mujer & 1,54 & 0,55 & & & \\
\hline \multirow{2}{*}{$\begin{array}{l}\text { Victimización } \\
\text { Verbal }\end{array}$} & Hombre & 1,96 & 0,67 & \multirow{2}{*}{0,221} & \multirow{2}{*}{0,638} & \multirow{2}{*}{0,696} \\
\hline & Mujer & 1,94 & 0,67 & & & \\
\hline \multirow{2}{*}{ Videojuegos } & Hombre & 36,11 & 7,31 & \multirow{2}{*}{4,016} & \multirow{2}{*}{0,046} & \multirow{2}{*}{0,001} \\
\hline & Mujer & 33,82 & 6,78 & & & \\
\hline \multirow{2}{*}{ Televisión } & Hombre & 2,94 & 1,30 & \multirow{2}{*}{7,477} & \multirow{2}{*}{0,006} & \multirow{2}{*}{0,044} \\
\hline & Mujer & 2,72 & 1,18 & & & \\
\hline \multirow{2}{*}{ Sustitución } & Hombre & 5,95 & 1,95 & \multirow{2}{*}{0,135} & \multirow{2}{*}{0,714} & \multirow{2}{*}{0,985} \\
\hline & Mujer & 5,95 & 3,55 & & & \\
\hline \multirow{2}{*}{ Irritabilidad } & Hombre & 7,05 & 2,45 & \multirow{2}{*}{37,334} & \multirow{2}{*}{0,000} & \multirow{2}{*}{0,001} \\
\hline & Mujer & 6,40 & 2,20 & & & \\
\hline
\end{tabular}

Se obtuvo un buen ajuste en todos los índices de evaluación del modelo de ecuaciones estructurales. El Chi-cuadrado reveló un valor significativo de $\mathrm{p}\left(\chi^{2}=\right.$ 31,286; gl = 8; p < 0,001), aunque debe considerarse que este índice no presenta límite superior. Por ello, no puede interpretarse de forma estandarizada, además de la problemática vinculada a su sensibilidad hacia tamaño muestral. En este sentido, se emplean varios índices de ajuste estandarizados que ofrecen una menor sensibilidad al tamaño muestral. El índice de ajuste comparativo (CFI) mostró un valor de 0,978, siendo excelente. El índice de ajuste normalizado (NFI) concretó un valor de 0,970 y el índice de incremento de ajuste (IFI) de 0,978, ambos excelentes. El error cuadrático medio de aproximación (RMSEA) refleja un valor adecuado de 0,075.

En la Tabla 3 y Figura 2 se observan los valores estimados de los parámetros del modelo. Estos deben presentar una magnitud adecuada y que los efectos sean significativamente distintos de cero. Asimismo, no deben presentarse estimaciones impropias como varianzas negativas.

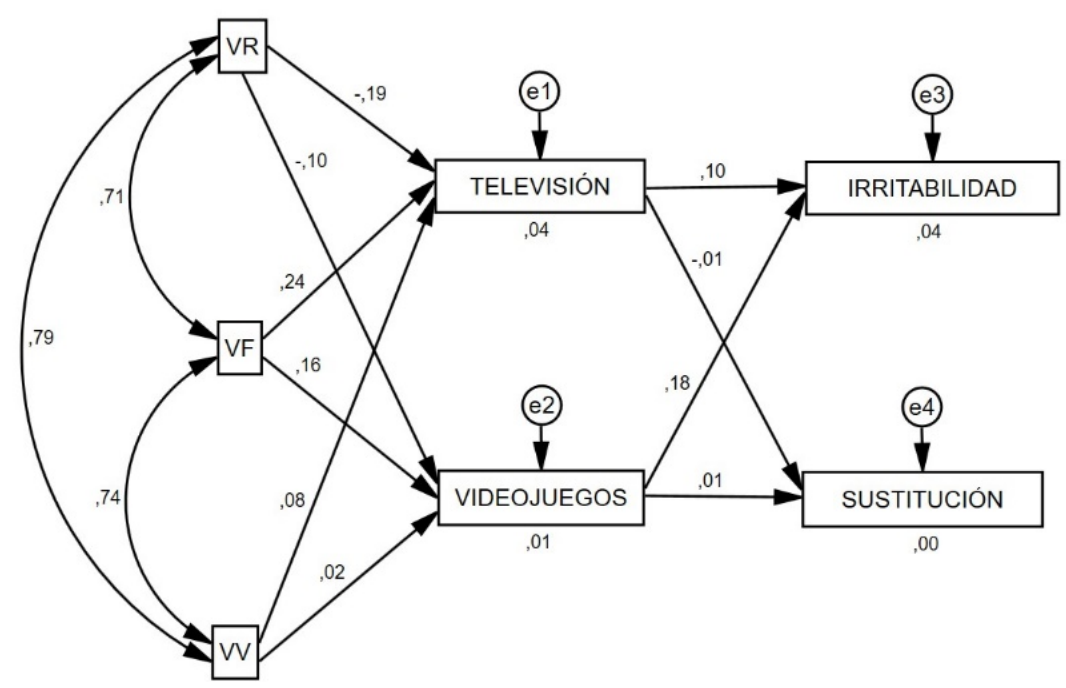

Figura 2. Modelo de ecuaciones estructurales de victimización y su relación con el uso de videojuegos y televisión 
Chacón-Cuberos, Ramón; Castro-Sánchez, Manuel; González-Campos, Gloria \& Zurita-Ortega, Félix (2018).

Victimización en la escuela, ocio digital e irritabilidad: análisis mediante ecuaciones estructurales. RELIEVE, 24(1), art. 3. doi: http://doi.org/10.7203/relieve.24.1.12614

Tabla 3 - Pesos de regresión y pesos estandarizados de regresión

\begin{tabular}{|c|c|c|c|c|c|c|c|}
\hline \multirow{2}{*}{\multicolumn{3}{|c|}{ Relaciones entre variables }} & \multicolumn{4}{|c|}{ P.R. } & \multirow{3}{*}{$\begin{array}{c}\text { P.E.R. } \\
\text { EST } \\
0,159\end{array}$} \\
\hline & & & \multirow{2}{*}{$\begin{array}{c}\text { EST } \\
0,180\end{array}$} & \multirow{2}{*}{$\begin{array}{l}\text { S.E. } \\
0,077\end{array}$} & \multirow{2}{*}{\begin{tabular}{|c|} 
C.R. \\
2,350
\end{tabular}} & \multirow{2}{*}{$\begin{array}{l}\mathbf{P} \\
*\end{array}$} & \\
\hline VIDEOJUEGOS & $\leftarrow$ & VF & & & & & \\
\hline VIDEOJUEGOS & & VR & $-0,119$ & 0,089 & $-1,335$ & 0,182 & $-0,101$ \\
\hline VIDEOJUEGOS & & VV & 0,020 & 0,082 & 0,249 & 0,804 & 0,020 \\
\hline TELEVISIÓN & $\leftarrow$ & VV & 0,074 & 0,072 & 1,028 & 0,304 & 0,080 \\
\hline TELEVISIÓN & & VR & $-0,200$ & 0,079 & $-2,542$ & $*$ & $-0,189$ \\
\hline TELEVISIÓN & $\leftarrow$ & VF & 0,244 & 0,068 & 3,595 & $* * *$ & 0,240 \\
\hline SUSTITUCION & & TELEVISIÓN & $-0,010$ & 0,040 & $-0,235$ & 0,814 & $-0,010$ \\
\hline SUSTITUCION & & VIDEOJUEGOS & 0,007 & 0,036 & 0,205 & 0,838 & 0,009 \\
\hline IRRITABILIDAD & & TELEVISIÓN & 0,077 & 0,033 & 2,359 & $*$ & 0,101 \\
\hline IRRITABILIDAD & & VIDEOJUEGOS & 0,123 & 0,029 & 4,197 & $* * *$ & 0,180 \\
\hline VR & & VV & 0,315 & 0,022 & 14,160 & $* * *$ & 0,795 \\
\hline VR & & VF & 0,258 & 0,020 & 13,173 & $* * *$ & 0,710 \\
\hline VV & $\leftrightarrow$ & VF & 0,304 & 0,022 & 13,507 & $* * *$ & 0,737 \\
\hline
\end{tabular}

${ }^{1}$ P.R., Pesos de Regresión; P.E.R., Pesos Estandarizados de Regresión; EST, Estimaciones; S.E., Estimación del error; C.R., Ratio Crítico.

${ }^{2}$ VF, Victimización Física; VR, Victimización Relacional; VV, Victimización Verbal

$3 *$ Relación entre variables estadísticamente significativa al nivel 0,05.

$4 * * *$ Relación entre variables estadísticamente significativa al nivel 0,005.

Se observan asociaciones estadísticamente significativas al nivel 0,005 entre todas las dimensiones de victimización, siendo todas ellas positivas y directas. Se muestra una relación directa en el uso de televisión con la victimización física ( $p<0,005 ; r=0,240)$, mientras que la asociación es inversa con la victimización relacional ( $\mathrm{p}<0,05 ; \mathrm{r}=$ $0,189)$. Asimismo, se observan relaciones estadísticamente significativas entre victimización física y videojuegos ( $\mathrm{p}<0,05$; $r=0,159)$, el uso de televisión y la irritabilidad ( $\mathrm{p}<0,05 ; \mathrm{r}=0,101)$ y el uso de videojuegos con esta misma variable ( $\mathrm{p}<$ $0,05 ; r=0,180)$, todas ellas positivamente relacionadas. Destacar que no se observaron diferencias estadísticamente significativas en las relaciones dadas entre videojuegos $\mathrm{y}$ victimización relacional y verbal, así como la sustitución de actividades con el uso de televisión y videojuegos. En los casos en los que no se encuentran asociaciones estadísticas se obtienen valores inferiores a 2 en el ratio crítico (C.R.), ya que los superiores suponen que el parámetro es diferente de 0 de forma estadísticamente significativa (Byrne, 2013). De este modo, puede establecerse que las escalas empleadas en las variables mencionadas no presentan validez convergente.

\section{Discusión}

Este trabajo de investigación aborda el modelo de multivictimización entre pares compuesto por tres dimensiones victimización relacional, verbal y físicapropuesto por Mynard y Joseph (2000) en estudiantes de tercer ciclo de Educación Primaria, así como su relación con el uso abusivo de dispositivos de ocio digital sedentario -televisión y videojuegos-. El modelo estructural desarrollado se ajustó de forma eficaz, tal y como demuestran estudios similares en el contexto nacional e internacional (Delisi et al., 2013; Espejo, Chacón, Zurita, \& Castro, 2016; Felix, Sharkey, Green, Furlong, \& Tanigawa, 2011; Shaw, Dooley, Cross, Zubrick, \& Waters, 2013; Vilches, 2015).

En primer lugar, y sobre los descriptivos básicos de la muestra de estudio, pudo observarse que los varones sufrían en mayor medida victimización física que las chicas, sin encontrar asociaciones estadísticamente 
significativas para la victimización física y verbal. Estos hallazgos pueden ser justificados por Ferguson (2015), quien señala las agresiones de tipo físico entre pares de sexo masculino como un medio para reforzar la autoestima, gestionar emociones negativas o incluso socializar. Asimismo, los niños veían más la televisión y jugaban más a los videojuegos que las niñas, datos que concuerdan con lo mostrado por Boxer et al. (2015), quienes atribuyen esta tendencia a la mayor existencia de dispositivos con un contenido que culturalmente ha sido asociado al género masculino. Por otro lado, el mayor uso de dispositivos digitales por parte de varones puede ser justificado también por una mayor búsqueda de recompensas externas a través del reforzamiento-recompensa que brinda el videojuego, lo que podría fundamentar los mayores niveles de irritabilidad encontrados para el género masculino (Chacón et al., 2017; Ditrrick et al., 2013).

En relación al modelo planteado, se observa que todas las dimensiones de victimización se relacionan entre ellas, revelando una asociación positiva y directa. Estudios como el de Cava, Buelga, Musitu y Murgui (2010) muestran el efecto negativo que producen las situaciones de acoso escolar en el ajuste psicosocial de las víctimas, revelando como estos efectos se relacionan principalmente con la presencia simultánea de agresiones de tipo directo e indirecto victimización física o verbal y victimización relacional-, lo que justificaría la relación positiva entre los tres tipos de victimización hallada, ya que estas tres dimensiones están interconectadas. De hecho, Shaw et al. (2013) y Vilches (2015) establecen como las agresiones físicas suelen ir acompañadas de acoso verbal, y estos dos tipos de conductas violentas, de acoso relacional o indirecto mediante el aislamiento o la influencia entre pares.

Los hallazgos muestran como la victimización física y el uso de televisión y videojuegos se asocian de forma directa. En estudios similares, Lam, Cheng y Liu (2013) analizaron las asociaciones existentes entre la exposición a videojuegos de contenido violento, victimización y ciberbullying, obteniendo que aquellos estudiantes que habían sufrido situaciones de acoso se exponían el doble a videojuegos violentos. Del mismo modo, Delisi et al. (2013), en un estudio sobre la influencia de videojuegos de contenido violento en la delincuencia y violencia juvenil, demostraron como este tipo de ocio digital afecta a variables psicológicas como los pensamientos, sentimientos y patrones de comportamiento violentos bien establecidos. Estos autores establecen que otros indicadores de antisocialidad pueden influir en la asociación entre exposición a videojuegos violentos y comportamientos violentos. No obstante, es una realidad que el uso de estos dispositivos constituye un factor de riesgo en esta problemática, afectando a la percepción del miedo, la ansiedad y la capacidad empática (Delisi et al., 2013; Ferguson et al., 2010; Méndiz, De Aguilera, \& Borges, 2011).

Del mismo modo, la victimización verbal se relacionó positivamente con el nivel de uso de televisión, la cual puede actuar como factor de riesgo por la exposición a contenido violento o bien como medio de evasión ante proceso de acoso escolar. Autores como Hiduja y Patchin (2010) o Watt et al. (2015) determinan como el abuso de televisión por parte de escolares se asocia con un aumento del riesgo de padecer victimización, relacionándose con problemas mentales, depresión, baja autoestima y rendimiento escolar. De hecho, García et al. (2010) y López y Sabater (2014) recuerdan cómo el ocio digital de pantalla ofrece a los sectores más jóvenes de población modelos de aprendizaje por observación de modelos cercanos, haciendo que niños y adolescentes aprendan conductas agresivas en sus diferentes manifestaciones. Dado el doble rol del agresor-víctima (Estévez, Jiménez, \& Moreno, 2010), estas premisas podrían justificar por qué los escolares que ven más televisión son los que reciben más victimización verbal, especialmente si obtienen beneficios a través de ella como 
evitación de agresiones o popularidad (Espejo et al., 2016; Ttofi \& Farrington, 2011).

Se reveló una relación positiva entre el uso de videojuegos y televisión y el nivel de irritabilidad en caso de no poder utilizarlos. Trabajos como los realizados por Carbonell (2014) y Van Rooij et al. (2010) destacan el número de horas empleadas como elemento diferenciador en el uso patológico y abusivo de dispositivos de pantalla, vinculándose con consecuencias de tipo social -como la concreción de relaciones sociales pobres, menor capacidad empática o bajo rendimiento académico-, y de tipo psicológico -manifestada mediante pulsión irresistible, focalización atencional, poca capacidad de control y estados de ánimo inestables (Boxer et al., 2015; Hasan et al., 2013)-, lo cual justifica los resultados obtenidos. Asimismo, es importante destacar la ausencia de relación entre el uso de televisión y videojuegos y la sustitución de actividades, lo cual puede deberse a la baja edad de la muestra de estudio, en la cual existe un mayor control parental en torno al tiempo de exposición y el contenido del ocio digital de pantalla (Vallejos \& Capa, 2010). De hecho, Both y Dunn (2013) y Macias, Gordillo y Camacho (2012) destacan la importancia del sector parental en la realización de tareas académicas en edades tempranas y en el desarrollo de hábitos saludables como el ocio deportivo (Garrido, Romero, Ortega \& Zagalaz, 2011), los cuales actúan como factores de prevención ante el uso patológico de estos dispositivos.

Finalmente, resulta de interés señalar las limitaciones que se vinculan a este estudio. La primera hace referencia al diseño de tipo descriptivo y corte transversal, el cual no permite desarrollar relaciones causales, algo que si permitiría un estudio longitudinal. También destacar la muestra de estudio, la cual ha estado compuesta por estudiantes de Educación Primaria, etapa en la que las situaciones de victimización no son tan asiduas como en la adolescencia, siendo de interés ampliar la muestra a la enseñanza secundaria en estudios futuros. Del mismo modo, hubiese resultado interesante la inclusión de otras variables en el estudio como son los tipos de conductas violentas, con el fin de definir el rol agresor-victima en relación al uso de videojuegos y televisión.

Las conclusiones alcanzadas en este estudio señalan un buen ajuste del modelo estructural desarrollado, así como una fiabilidad aceptable. Este estudio genera una visión concreta sobre las relaciones existentes entre los diferentes tipos de victimización que se producen en el contexto escolar y el uso abusivo de medios de ocio digital. Se obtuvieron relaciones positivas entre los tres tipos de victimización, la victimización física y el uso de videojuegos y televisión, y la victimización verbal con el uso de televisión. Del mismo modo, ambos hábitos de ocio digital se asociaron positivamente con los niveles de irritabilidad de los escolares. En este sentido, se muestra que las situaciones de acoso escolar pueden actuar como un factor de riesgo en el uso problemático de videojuegos y televisión, hábitos que pueden ocasionar efectos negativos a nivel cognitivo y socio-afectivo, especialmente en el desarrollo de niveles concretos de agresividad y pobres habilidades sociales. Por tanto, se destaca la importancia de que el sector parental regule la exposición al ocio de pantalla de los niños y niñas en edad escolar.

\section{Referencias}

Benbenishty, R., Avi, R. Roziner, L. \& Wrabel, S. (2016). Testing the Causal Links between School Climate, School Violence, and School Academic Performance. A Cross-Lagged Panel Autoregressive Model. Educational Researcher, 45(3), 197-206. doi: https://doi.org/10.3102/0013189X16644603

Booth, A. \& Dunn, J. F. (2013). Family-school links: How do they affect educational outcomes? Routledge: New York.

Boxer, P., Groves, C., \& Docherty, M. (2015). Video games do indeed influence children and adolescents' aggression, prosocial behaviour, and academic performance. A clearer reading of Ferguson (2015). Perspectives on 
Psychological Science, 10(5), 671-673. doi: https://doi.org/10.1177/1745691615592239

Byrne, B. M. (2013). Structural equation modelling with AMOS: Basic concepts, applications, and programming. Routledge: New York.

Carbonell, X. (2014). La adicción a los videojuegos en el DSM-5. Adicciones, 26(2), 91-95.

doi:

https://doi.org/10.20882/adicciones.10

Carrasco, C. \& Trianes, M. V. (2015). Clima social, prosocialidad y violencia como predictores de inadaptación escolar en primaria. European Journal of Education and Psychology, 3(2), 229-242. doi: 10.1989/ejep.v2i2.62.

Cava, M. J., Buelga, S., Musitu, G., \& Murgui, S. (2010). Violencia escolar entre adolescentes y sus implicaciones en el ajuste psicosocial: un estudio longitudinal. Revista de Psicodidáctica, 15(1), 21-34. doi: https://doi.org/10.1387/RevPsicodidact.732

Cava, M. J., Musitu, G., \& Murgui, S. (2007). Individual and social risk factors related to overt victimization in a sample of Spanish adolescents. Psychological Reports, 101(1), 275-290.

https://doi.org/10.2466/pr0.101.1.275-290

Chacón, R., Castro, M., Zurita, F., Espejo, T., \& Martínez, A. (2016). Videojuegos activos como recurso TIC en el aula de Educación Física: estudio a partir de parámetros de ocio digital. Digital Education Review, 29, 112123.

Chacón, R., Zurita, F., Castro, M., Espejo, T., Martínez, A., \& Pérez, A. J. (2017). Motivational climate in sport and its relationship with digital sedentary leisure habits in university students. Saúde $e$ Sociedade, 26(1), 29-39. doi: https://doi.org/10.1590/s0104$\underline{12902017166561}$

Chamarro, A., Carbonell, X., Manresa, J. M., Muñoz-Mirallles, R., Ortega-González, R., López-Morrón, M. R., Batalla-Martínez, C. \& Torán-Monserrat, P. (2014). El Cuestionario de Experiencias Relacionadas con los Videojuegos (CERV): Un instrumento para detectar el uso problemático de videojuegos en adolescentes españoles. Adicciones, 26(4), 303-311. doi: https://doi.org/10.20882/adicciones.31

Delisi, M., Vaighn, M. G., Gentile, D. A., Anderson, C. A., y Shook, J. J. (2013). Violent video games, delinquency, and youth violence new evidence. Youth Violence and Juvenile Justice, 11(2), 132-142. doi: https://doi.org/10.1177/1541204012460874

Ditrrick, C., Beran, T., Mishna, F., Hetherington, R., \& Shariff, S. (2013). Do children who bully their peers also play violent video games? A Canadian national study. Journal of School Violence, 12(4), 297-318. doi:

https://doi.org/10.1080/15388220.2013.803244

Espejo, T., Chacón, R., Zurita, F., \& Castro, M. (2016). Victimización en edad escolar desde la perspectiva de la actividad física. Sportis, 2(3), 379-389.

doi:

https://doi.org/10.17979/sportis.2016.2.3.1729

Estévez, E., Jiménez, T. I., \& Moreno, D. (2010). Cuando las víctimas de violencia escolar se convierten en agresores: ¿Quién va a defenderme? European Journal of Education and Psychology, 3(2), 177-186. doi: https://doi.org/10.1989/ejep.v3i2.58

Felix, E., Sharkey, J., Green, J., Furlong, M., \& Tanigawa, D. (2011). Getting precise and pragmatic about the assessment of bullying: The development of the California Bullying Victimization Scale. Aggressive Behavior, 37(3), 234-247. doi: https://doi.org/10.1002/ab.20389

Ferguson, C. J. (2015). Do angry birds make for angry children? A meta-analysis of video game influences on children's and adolescents' aggression, mental health, prosocial behavior, and academic performance. Perspectives on Psychological Science, 10(5), 646-666. doi: https://doi.org/10.1177/1745691615592234

Ferguson, C., Olson, C., Kutner, L., \& Warner, D. (2010). Violent video games, catharsis seeking, bullying and delinquency: a multivariate analysis of effects. Crime $y$ Delinquency, 4, 1-21. doi: https://doi.org/10.1177/0011128710362201

García, X., Pérez, A., \& Nebot, M. (2010). Factores relacionados con el acoso escolar 
(bullying) en los adolescentes de Barcelona. Gaceta Sanitaria, 24(2), 103-108. doi: https://doi.org/10.1016/j.gaceta.2009.09.017

Garrido, M. E., Romero, S., Ortega, E., \& Zagalaz, M. L. (2011). Designing a questionnaire of parents for children sport. Journal of Sport and Health Research, 3(1), 59-70.

Hasan, Y., Bègue, L., Scharkow, M., \& Bushman, B. J. (2013). The more you play, the more aggressive you become: A long-term experimental study of cumulative violent video game effects on hostile expectations and aggressive behaviour. Journal of Experimental Social Psychology, 49(2), 224-227. doi: https://doi.org/10.1016/j.jesp.2012.10.016

Hiduja, S. \& Patchin, J. W. (2010). Bullying, cyberbullying, and suicide. Archives of Suicide Research, 14(3), 206-221. doi: https://doi.org/10.1080/1381118.2010.494133

Lam, L., Cheng, Z., \& Liu, X. (2013). Violent online games exposure and cyberbullying/victimization among adolescents. Cyberpsychology, Behavior, and Social Networking, 16(3), 159-165. doi: https://doi.org/10.1089/cyber.2012.0087

López, L. \& Sabater, C. (2014). Medios audiovisuales y acoso escolar: buenas prácticas para la prevención y promoción de la convivencia. Revista de Investigación en Educación, 12(2), 145-163.

Macias, A., Gordillo, L., \& Camacho, E. (2012). Hábitos alimentarios de niños en edad escolar y el papel de la educación para la salud. Revista Chilena de Nutrición, 39(3), 40-43. doi: $\quad$ https://doi.org/10.4067/S071775182012000300006

Martínez, P., Ocampo, D., \& González, A. (2013). Uso de videojuegos, agresión, sintomatología depresiva y violencia intrafamiliar en adolescentes $\mathrm{y}$ adultos jóvenes. Revista Colombiana de Ciencias Sociales, 4(2), 167-180. doi: https://doi.org/10.21501/22161201.862

Marsh, H. W. (2007). Handbook of Sport Psychology. Third Edition. New Jersey: Tenenbaum and R. C. Eklund. doi: https://doi.org/10.1002/9781118270011.ch35
Méndiz, A., De Aguilera, M., \& Borges, E. (2011). Actitudes y valoraciones de los jóvenes ante la TV móvil. Comunicar, 36(1), 77-85. doi: https://doi.org/10.3916/C36-2011$\underline{02-08}$

Mishna, F., Khoury-Kassabri, M., Gadalla, T., \& Daciuk, J. (2012). Risk factors for involvement in cyber bullying: Victims, bullies and bully-victims. Children and Youth Services Review, 34(1), 63-70. doi: https://doi.org/10.1016/j.childyouth.2011.08.0 $\underline{32}$

Mynard, H. \& Joseph, S. (2000). Development of the Multidimensional Peer-Victimization Scale. Aggressive Behavior, 26(1), 169-178. doi: $\quad$ https://doi.org/10.1002/SICI109823372000262

Shaw, T., Dooley, J. J., Cross, D., Zubrick, S. R., \& Waters, S. (2013). The forms of bullying scale (FBS): Validity and reliability estimates for a measure of bullying victimization and perpetration in adolescence. Psychological Assessment, 25(4), 1045-1057. doi: https://doi.org/10.1037/a0032955

Ttofi, M. \& Farrington, D. (2011). Effectiveness of school-based programs to reduce bullying: A systematic and meta-analytic review. Journal of Experimental Criminology, 7(1), 27-56. doi: https://doi.org/10.1007/s11292$\underline{010-9109-1}$

Turner, H. A., Shattuck, A., Finkelhor, D., \& Hamby, S. (2017). Effects of PolyVictimization on Adolescent Social Support, Self-Concept and Psychologial Distress. Journal of Interpersonal Violence, 32(5), 755780.

doi: https://doi.org/10.1177/08862605155586376

Turner, M. G., Exum, M. L., Brame, R., \& Holt, T. J. (2013). Bullying victimization and adolescent mental health: General and typological effects across sex. Journal of Criminal Justice, 41(1), 53-59. doi: https://doi.org/10.1016/j.jcrimjus.2012.12.005

Vallejos, M. \& Capa, W. (2010). Videojuegos: Adicción y factores predictores. Avances en Psicología, 18(1), 103-110.

Van Rooij, A. J., Schoenmakers, T. M., Vermulst, A., Vermulst, A., Van den Eijden, R., \& Van de Mheen, D. (2010). Online video 
Chacón-Cuberos, Ramón; Castro-Sánchez, Manuel; González-Campos, Gloria \& Zurita-Ortega, Félix (2018).

Victimización en la escuela, ocio digital e irritabilidad: análisis mediante ecuaciones estructurales. RELIEVE, 24(1), art.

3. doi: http://doi.org/10.7203/relieve.24.1.12614

game addiction: identification of addicted adolescent gamers. Addiction, 106, (1), 205210. doi: https://doi.org/10.1111/j.13600443.2010.03104.x

Vilches, J. M. (2015). Centros especializados y normalizados de secundaria: relación entre autoestima, agresividad, victimización $y$ calidad de vida en estudiantes de Granada capital. Tesis Doctoral: Universidad de Granada.
Watt, E., Fitzpatrick, C., Derevensky, J., \& Pagani, L. (2015). Too much television? Prospective associations between early childhood televiewing and later self-reports of victimization by sixth grade classmates. Journal of Developmental y Behavioral Pediatrics, 36(6), 426-433. doi: https://doi.org/10.1097/DBP.00000000000001 $\underline{86}$

\begin{tabular}{|c|c|}
\hline $\begin{array}{l}\text { Authors / Autores } \\
\end{array}$ & $\begin{array}{l}\text { To know more } \\
\text { / Saber más }\end{array}$ \\
\hline \multirow{4}{*}{$\begin{array}{l}\text { Chacón-Cuberos, Ramón (rchacón@ugr.es) } \\
\text { Departamento de Didácticas Integradas. Universidad de Huelva. Su dirección } \\
\text { postal es Facultad de Ciencias de la Educación. Avenida de las Fuerzas Armadas, } \\
\text { S/N. } 21071 \text { Huelva (España) }\end{array}$} & ORCID \\
\hline & 0000-0003-0937-1089 \\
\hline & Google \\
\hline & ResearchGate \\
\hline \multirow{4}{*}{$\begin{array}{l}\text { Castro-Sánchez, Manuel (mcastros@ual.es). } \\
\text { Profesor del área de conocimiento de Didáctica de la Expresión Corporal en el } \\
\text { Departamento de Educación de la Universidad de Almería (España). Es el autor } \\
\text { de contacto para este artículo. Dirección Postal: Universidad de Almería, } \\
\text { Departamento de Educación. Edificio Central, Planta 1. Ctra Sacramento, s/n. } \\
04120 \text { - La Cañada, Almería (España). }\end{array}$} & ORCID \\
\hline & $0002-2357-3093$ \\
\hline & ResearchGate \\
\hline & \\
\hline \multirow{2}{*}{$\begin{array}{l}\text { González-Campos, Gloria (gloriagc@us.es). } \\
\text { Departamento de Didáctica de la Expresión Corporal. Universidad de Sevilla }\end{array}$} & \\
\hline & \\
\hline \multirow{4}{*}{$\begin{array}{l}\text { Zurita-Ortega, Félix (felixzo@ugr.es) } \\
\text { Profesor Titular del Departamento de Didáctica de la Expresión Musical, } \\
\text { Plástica y Corporal de la de la Facultad de Ciencias de la Educación de la } \\
\text { Universidad de Granada. Su dirección postal es: Facultad de Ciencias de la } \\
\text { Educación. Campus Universitario de Cartuja, s/n. C.P. } 18071 \text { - Granada (España). }\end{array}$} & ORCID \\
\hline & 0000-0002-1189-894X \\
\hline & \\
\hline & \\
\hline
\end{tabular}

\section{RELIEVE}

Revista ELectrónica de Investigación y EValuación Educativa E-Journal of Educational Research, Assessment and Evaluation

[ISSN: 1134-4032]

(C) Copyright, RELIEVE. Reproduction and distribution of this articles it is authorized if the content is no modified and their origin is indicated (RELIEVE Journal, volume, number and electronic address of the document).

(C) Copyright, RELIEVE. Se autoriza la reproducción y distribución de este artículo siempre que no se modifique el contenido y se indique su origen (RELIEVE, volumen, número y dirección electrónica del documento). 